\title{
Joint Communication and Control System Design for Connected and Autonomous Vehicle Navigation
}

\author{
Tengchan Zeng*, Omid Semiari ${ }^{\dagger}$, Walid Saad*, and Mehdi Bennis ${ }^{\ddagger}$ \\ *Wireless@VT, Bradley Department of Electrical and Computer Engineering, Virginia Tech, Blacksburg, VA, USA \\ ${ }^{\dagger}$ Department of Electrical and Computer Engineering, Georgia Southern University, Statesboro, GA, USA \\ ${ }^{\ddagger}$ Centre for Wireless Communications, University of Oulu, Oulu, Finland \\ Emails: * ${ }^{*}$ tengchan, walids $\} @$ vt.edu, ${ }^{\dagger}$ osemiari@ georgiasouthern.edu, ${ }^{\ddagger}$ mehdi.bennis@ oulu.fi
}

\begin{abstract}
Connected and autonomous vehicles (CAVs) are able to improve on-road safety and provide convenience in our daily lives. To perform autonomous path tracking and navigation, CAVs can exploit vehicle-to-everything (V2X) communications to determine their vehicle dynamics parameters, such as location, heading angle, and curvature, which can be then used as inputs to their control system. However, the interference and uncertainty of the wireless channels can increase the transmission delay on the vehicle dynamics and, thus, impair the CAV's ability to track its target path. In this paper, the problem of joint communication network and control system design is studied to solve the path tracking problem for CAVs. In particular, a novel approach is proposed to maximize the number of reliable $\mathrm{V} 2 \mathrm{X}$ transmitter-receiver pairs while jointly considering the stability of the controller and the state of the wireless network. Based on the joint design, the maximum transmission delay which can prevent instability in the controller is determined. Then, the reliable V2X links maximization problem is decomposed into two equivalent sub-problems. The first sub-problem is the control mechanism design in which a dual update method is used to determine the headway distance parameter for the control system. The second sub-problem uses the outcome of the first sub-problem to optimize the power allocation for the communication system. To solve this power allocation problem, a novel risk-based approach that uses the so-called conditional value at risk (CVaR) from financial engineering is proposed. Simulation results validate the theoretical results and show that the proposed joint design can improve the number of reliable V2X pairs by as much as $70 \%$ compared to a baseline scheme that optimizes the communication and control systems independently.
\end{abstract}

\section{INTRODUCTION}

Connected and autonomous vehicles (CAVs) will be a pillar of tomorrow's intelligent transportation systems (ITSs) [1]. To operate effectively, CAVs must sense and track the environment to determine their navigation path. In order to obtain the information needed for their navigation, CAVs must leverage wireless connectivity by using vehicle-to-everything (V2X) communications. However, integrating CAVs into 5G-enabled V2X networks requires overcoming several challenges, including joint communication and control [2], network capacity [3], and network modeling [4].

In particular, to perform autonomous navigation, the CAVs must follow a predesigned path by constantly adjusting their control system whose inputs are the CAV dynamics, such as location, heading angle and curvature. The CAV's controller can use the difference between the current parameters of the vehicle dynamics and the target ones as a feedback signal

This research was supported, in part, by the U.S. National Science Foundation under Grants CNS-1739642 and CNS-1836802, by the Academy of Finland Project CARMA, by the Academy of Finland Project MISSION, by the Academy of Finland Project SMARTER, as well as by the INFOTECH Project NOOR. to make appropriate adjustments to the steering direction and velocity. Such vehicle dynamics are typically measured by sensors [4] or estimated by algorithms, such as map matching [5]. However, the data collected from sensors is not always accurate to capture the dynamics of CAVs. For example, in an urban scenario, the global positioning system (GPS) signal can be easily blocked by surrounding buildings and the location error estimated by GPS sensor can be as high as ten meters [6]. Also, due to the complexity of the estimation algorithms, inferring a CAV's dynamics can be time-consuming, leading to a potential safety risk to the real-time vehicular system operation. In practice, sensor data can be complemented by measurements received from V2X communications [1].

In fact, the works in [6]-[9] have demonstrated that V2X communications can yield very accurate vehicle dynamics information. For example, in [6] and [7], the authors propose to use both GPS data and information received from vehicleto-vehicle $(\mathrm{V} 2 \mathrm{~V})$ communications to improve the accuracy of location. Moreover, the authors in [8] utilize vehicle-toinfrastructure (V2I) communications to inform vehicles of their geographic locations and lane numbers in an intersection scenario. In addition, the work in [9] leverages multihop V2X communications to aid vehicles blocked by buildings to estimate their locations. While the information collected from V2X communications can help improve the accuracy of CAVs' dynamics, the information transmitted via V2X communications will be inevitably coupled with the transmission delay. For CAVs, such delayed information can lead to instability of the controller thus preventing proper navigation by the CAVs [10]. To better leverage V2X links for autonomous navigation and path tracking, there is a need to determine how the delayed information impacts the stability of the controller. To do so, one must jointly design the communication and control system of a CAV. In this regard, none of the prior works [6]-[11] studied this joint communication and control design problem in $5 \mathrm{G}$ cellular V2X systems, as they often assumed the control or communication system to be a blackbox.

The main contribution of this paper is a novel joint control system and V2X communication design framework that enables CAVs to leverage V2X connectivity for autonomous navigation (i.e., path tracking). In particular, we first analyze two typical road scenarios for path tracking, and, then, we analytically determine the maximum time delay to guarantee the stability of the controller in these two scenarios. Using this analysis, we optimize the control system design and the power allocation for the communication network so as to maximize the number of vehicular communication links that meet the 
delay requirements. To the best of our knowledge, this is the first work that considers the impact of the transmission delay in $5 G$ networks on the stability of the path tracking controller while jointly designing the control and communication system for wireless-enabled autonomous vehicle navigation. Simulation results validate the theoretical results and show that the proposed joint design can improve the number of reliable V2X pairs by as much as $50 \%$ compared to a baseline that optimizes the communication and control systems independently.

\section{SySTEM MOdEL}

Consider a V2X system that follows the Manhattan mobility model [12] and includes CAVs driving on the road, pedestrians walking on the sidewalks, and base stations (BSs)/road side units (RSUs) along the roads. In this system, each CAV will receive information from nearby BSs/RSUs, pedestrians, or $\mathrm{CAV}$ s/wireless enabled normal vehicles via $\mathrm{V} 2 \mathrm{X}$ links to estimate parameters affecting its movements such as location, heading angle, or driving curvature. By using this information as input, the control system of each CAV can properly adjust its trajectory to follow the designed path.

\section{A. Communication Model}

We consider a set $\mathcal{I}$ of $I \mathrm{~V} 2 \mathrm{X}$ links, consisting of all $\mathrm{V} 2 \mathrm{~V}$ pairs, vehicle-to-pedestrian (V2P) pairs, and V2I pairs in the network, where the receiver in each link is a CAV and the transmitter can be a BS/RSU, pedestrian, or CAV. Also, we assume that the transmitter-receiver configuration of each V2X pair is fixed during the entire communication period, and the distance between the transmitter and receiver of each V2I pair is bounded within a safety range. We also consider a Cartesian coordinate system centered on an arbitrarily selected point, and the locations of the transmitter and receiving CAV in V2X link $i$ at time $t$ are denoted by, respectively, $\left(x_{i}(t), y_{i}(t)\right)$ and $\left(x_{i}^{\prime}(t), y_{i}^{\prime}(t)\right), i \in \mathcal{I}$. Due to the large number of CAVs and limited bandwidth, we assume that all V2X links share the same frequency resource, and, thus, each V2X link will suffer from the interference generated by other links.

To model the wide range of fading environment for vehicular networks, we consider the V2X channels as independent Nakagami channels [13]. Thus, the channel gain between the receiving CAV $i$ and the transmitter $j$ at slot $t$ will be $g_{i, j}(t)=h_{i, j}(t) l_{i, j}(t)$, where $h_{i, j}(t)$ captures the Nakagami fading channel gain, and $l_{i, j}(t)$ is the path loss. Moreover, the assigned transmission power $p_{i}(t)$ for $\mathrm{V} 2 \mathrm{X}$ link $i \in \mathcal{I}$ at time slot $t$ should satisfy $p_{i}(t) \leq p_{i}^{\max }$, where $p_{i}^{\max }$ denotes the maximum transmission power of the transmitter in V2X link $i$ and the values of $p_{i}^{\max }$ for vehicles, pedestrians, and BSs/RSUs are different. Hence, the throughput of V2X pair $i$ will be:

$$
R_{i}(t)=\omega \log _{2}\left(1+\frac{p_{i}(t) g_{i, i}(t)}{\omega N_{0}+\sum_{j \in \mathcal{I} \backslash i} p_{j}(t) g_{i, j}(t)}\right),
$$

where $\omega$ is the bandwidth of the shared channel, and $N_{0}$ is the noise power spectral density. Using (1), we can obtain the V2X link transmission delay as $\tau_{i}(t)=S / R_{i}(t)$, where $S$ is the size of the transmitted packet in bits.

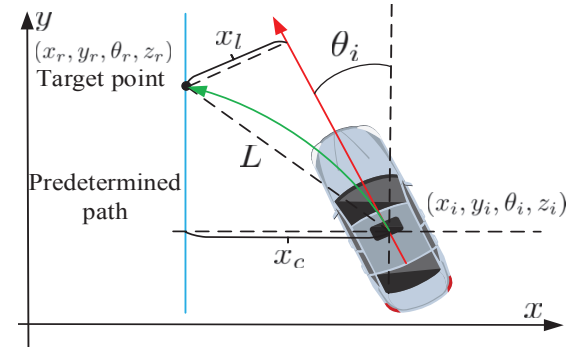

Fig. 1. Steering control for CAVs.

\section{B. Controller Model}

As shown in Fig. 1, consider the receiving CAV in V2X link $i$ as an example, and the parameters capturing vehicle dynamics include location $\left(x_{i}(t), y_{i}(t)\right)$, heading direction $\theta_{i}(t)$, and vehicle curvature $z_{i}(t)$. Note that $\theta_{i}(t)$ is taken counterclockwise from the $y$-axis. To track the predetermined navigation path, a CAV will typically adopt the pure pursuit method [14]. In this method, the steering controller in the CAV will constantly calculate the curvature of the circular arc that connects the current location to the target point on the reference trajectory ahead of the vehicle by the headway distance $L$, as shown in Fig. 1. Moreover, as path tracking has been usually studied for constant velocities [14], the goal of the longitudinal controller is to keep a constant speed $v$. Here, we assume the target point is located at $\left(x_{r}, y_{r}\right)$, and the associated heading orientation angle and the curvature are, respectively, $\theta_{r}$ and $z_{r}$. Thus, as shown in Fig. 1, we obtain the lateral position error $x_{l}(t)$ and the errors of the heading angle and vehicle's curvature can be expressed as

$$
\theta_{e}(t)=\theta_{i}(t)-\theta_{r}, z_{e}(t)=z_{i}(t)-z_{r} .
$$

Also, we capture the state representation of CAV $i$ by using the following differential equations [10]:

$$
\begin{aligned}
& \dot{x}_{i}(t)=-v \sin \left(\theta_{i}(t)\right), \\
& \dot{y}_{i}(t)=v \cos \left(\theta_{i}(t)\right), \\
& \dot{\theta}_{i}(t)=v z_{i}(t), \dot{z}_{i}(t)=\left(z\left(t-\tau_{i}(t)\right)-z_{i}(t)\right) / T,
\end{aligned}
$$

where $T$ is the duration for each time slot, and $z\left(t-\tau_{i}(t)\right)$ is the output of the steering controller with $\tau_{i}(t)$ being the transmission delay in V2X links. In addition, similar to [10] and [14], we assume that the steering controller output is determined by the lateral position error $x_{l}(t)$, heading angle error $\theta_{e}(t)$, and curvature error $z_{e}(t)$. As the vehicle dynamics information is obtained through V2X links, the errors $x_{l}(t)$, $\theta_{e}(t)$, and $z_{e}(t)$ will be impacted by the transmission delay.

\section{Problem Formulation}

As discussed in Section I, the delayed navigation information can lead to control system instability, meaning that the CAV's movements will not converge to the predetermined path [10]. Accordingly, we can define a reliability metric as the probability of the wireless network meeting the maximum delay requirement $\tau_{i}^{\max }, i \in \mathcal{I}$, to maintain the stability of the steering controller. The reliability of each V2X link can be thereby defined as $\operatorname{Pr}\left(\tau_{i} \leq \tau_{i}^{\max }\right), i \in \mathcal{I}$. There are two approaches to increasing the reliability. That is, when designing the wireless network, we can optimize the power allocation for $\mathrm{V} 2 \mathrm{X}$ links to increase the throughput and reduce the delay $\tau_{i}$. Also, when designing the control system, we 
can relax the maximum delay requirement $\tau_{i}^{\max }$ by choosing a proper value for the control system, such as the headway distance $L_{i}$ in the steering controller.

Different from the prior work in [2] which solely optimizes the design of the control system for a single vehicular platoon system, we propose to maximize the number of reliable V2X links for a set of independent CAVs by jointly designing the wireless network and the control system. The joint design can be posed as an optimization problem in which transmission power is allocated to each V2X link and the headway distance is selected so as to maximize the number of reliable V2X pairs. To this end, we can formulate the problem as

$$
\begin{aligned}
& \max _{\left\{L_{i}\right\}, \boldsymbol{p}} \sum_{i \in \mathcal{I}} \mathbb{1}\left(\operatorname{Pr}\left(\tau_{i}(t) \leq \tau_{i}^{\max }\right) \geq \gamma\right) \\
& \text { s.t. } 0 \leq p_{i}(t) \leq p_{i}^{\max }, i \in \mathcal{I}, \\
& \quad L_{\min } \leq L_{i} \leq L_{\max },
\end{aligned}
$$

where $\boldsymbol{p}=\left(p_{i}(t), i \in \mathcal{I}\right)$ is the transmission power vector for the V2X links in the network and $\gamma \in(0,1)$ is the minimum reliability requirement for reliable $\mathrm{V} 2 \mathrm{X}$ communication links. In particular, $\mathbb{1}\left(\operatorname{Pr}\left(\tau_{i}(t) \leq \tau_{i}^{\max }\right) \geq \gamma\right)=1$ when $\operatorname{Pr}\left(\tau_{i}(t) \leq\right.$ $\left.\tau_{i}^{\max }\right) \geq \gamma$; otherwise, $\mathbb{1}\left(\operatorname{Pr}\left(\tau_{i}(t) \leq \tau_{i}^{\max }\right) \geq \gamma\right)=0$. Constraint (7) ensures that the allocated power will not exceed the maximum transmission power and constraint (8) guarantees that the headway distance $L_{i}, i \in \mathcal{I}$, is selected within a reasonable range.

To solve the joint control and communication optimization problem in (6)-(8), we will first use the Lyapunov-Razumikhin theorem to derive the maximum V2X communication delay $\tau_{i}^{\max }$, which can guarantee the stability for the controller when the CAV tracks two different path types. As the delay requirement $\tau_{i}^{\max }$ is only dependent on $L_{i}, i \in \mathcal{I}$, and $\operatorname{Pr}\left(\tau_{i}(t) \leq \tau_{i}^{\max }\right)$ is an increasing function of $\tau_{i}^{\max }$, we can rewrite the objective function (6) as $\max _{\boldsymbol{p}} \sum_{i \in \mathcal{I}} \mathbb{1}\left(\operatorname{Pr}\left(\tau_{i}(t) \leq \max _{\left\{L_{i}\right\}} \tau_{i}^{\max }\right) \geq \gamma\right)$. In this case, we can decompose the optimization problem into two sub-problems where the optimal solution to these subproblems is equivalent to the one for the original problem. The first sub-problem seeks to find an appropriate value for the headway distance $L_{i}$ to $\operatorname{relax} \tau_{i}^{\max }, i \in \mathcal{I}$. Then, under an optimized $\tau_{i}^{\max }$, we leverage the conditional value at risk (CVaR) [15] from financial engineering to determine the power allocation strategy in the wireless system in order to maximize the number of reliable V2X links in the second sub-problem.

\section{Stability Analysis of the Control System}

As shown in [16], any path can be represented as a combination of straight lines and circular curves. Here, we conduct the stability analysis for the steering controller in which CAVs use information received from V2X links to track straight lines and circular curves. Based on the analysis, we also derive the maximum allowable communication delay in each scenario which can guarantee a stable operation for the CAV.

\section{A. Tracking Straight Lines}

As shown in Fig. 1, consider that the CAV follows a straight line and the $y$-axis is parallel to the straight line. In this case, we can obtain the heading error, the steering error, and the lateral position error, respectively, as $\theta_{e}(t)=\theta_{i}(t), z_{e}(t)=$ $z_{i}(t)$, and $x_{l}(t)=-\left[x_{e}(t) \cos \theta_{i}(t)-\sqrt{L_{i}^{2}-x_{e}(t)^{2}} \sin \theta_{i}(t)\right]$ with

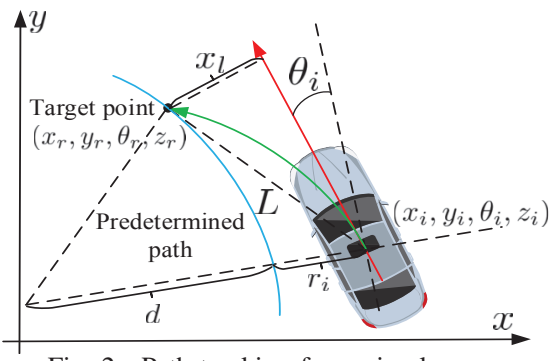

Fig. 2. Path tracking for a circular curve.

$x_{e}(t)=x(t)-x_{r}$. As the lateral position error is a function of $x_{e}(t)$ and does not depend on $y_{e}(t)=y(t)-y_{r}$, then (3) and (5) are sufficient to capture the state representation of the CAV. Moreover, when the vehicle is tracking a straight line, the output of the steering controller can be shown as [10]:

$$
z(t)=\frac{2}{L_{i}^{2}}\left(x_{i}(t) \cos \theta_{i}(t)-\sqrt{L_{i}^{2}-x_{i}^{2}(t)} \sin \theta_{i}(t)\right) .
$$

As observed in the state representations in (3) and (5), $\boldsymbol{e}(t)=\left(x_{i}(t), \theta_{i}(t), z_{i}(t)\right)$ is equal to $(0,0,0)$ when the vehicle reaches its destination. Thus, the state representation of the CAV can be linearized around $(0,0,0)$ as follows:

$$
\dot{e}(t)=\bar{A} \boldsymbol{e}(t)+\bar{B} \boldsymbol{e}(t-\tau),
$$

where the Jacobian matrices $\overline{\boldsymbol{A}}=\left[0,-v, 0 ; 0,0, v ; 0,0,-\frac{1}{T}\right]$ and $\overline{\boldsymbol{B}}=\left[0,0,0 ; 0,0,0 ; \frac{\phi_{x_{i}}}{T}, \frac{\phi_{\theta_{i}}}{T}, \frac{\phi_{z_{i}}}{T}\right]$ with $\phi_{x_{i}}=\left.\frac{\partial z}{\partial x_{i}}\right|_{(0,0,0)}=$ $\frac{2}{L_{i}^{2}}, \phi_{\theta_{i}}=\left.\frac{\partial z}{\partial \theta_{i}}\right|_{(0,0,0)}=\frac{-2}{L_{i}}$, and $\phi_{z_{i}}=\left.\frac{\partial z}{\partial z_{i}}\right|_{(0,0,0)}=0$.

\section{$B$. Tracking Circular Curves}

As shown in Fig. 2, consider that the CAV follows a curve with a constant curvature. By using polar coordinates, the state representation of the CAV can be captured as follows [10]:

$$
\begin{aligned}
& \dot{r}_{i}(t)=-v \sin \left(\theta_{i}(t)\right), \quad \dot{z}_{i}(t)=-\frac{z_{i}(t)+z_{r}}{T}+\frac{z(t-\tau)}{T}, \\
& \dot{\theta}_{i}(t)=v\left(z_{i}(t)+z_{r}-\frac{z_{r} \cos \theta_{i}(t)}{1+r(t) z_{r}}\right)
\end{aligned}
$$

where $r_{i}(t)$ is the radial distance from the path to the CAV, and $z_{r}=1 / d$ with the radius $d$ of the circular curve. According to [10], we can obtain the output of the steering controller as $z(t)=\frac{2}{L_{i}^{2}}\left[\hat{z}(t) \cos \theta_{i}(t)-\sqrt{L_{i}^{2}-\hat{z}(t)^{2}} \sin \theta_{i}(t)\right]$ with $\hat{z}(t)=$ $\frac{z_{r}\left(r_{i}(t)^{2}+L_{i}^{2}\right)+2 r_{i}(t)}{2\left(1+z_{r} r_{i}(t)\right)}$. When $\boldsymbol{e}(t)=(0,0,0)$, we can verify that the CAV reaches the destination. Thus, the state representation of the CAV can be linearized around $(0,0,0)$ as follows

$$
\dot{\boldsymbol{e}}(t)=\hat{\boldsymbol{A}} \boldsymbol{e}(t)+\hat{\boldsymbol{B}} \boldsymbol{e}(t-\tau) \text {, }
$$

where the Jacobian matrices $\hat{\boldsymbol{A}}=\left[0,-v, 0 ; v z_{r}^{2}, 0, v ; 0,0,-\frac{1}{T}\right]$ and $\hat{\boldsymbol{B}}=\left[0,0,0 ; 0,0,0 ; \frac{\varphi_{r}}{T}, \frac{\varphi_{\theta_{i}}}{T}, \frac{\varphi_{z_{i}}}{T}\right]$ with $\varphi_{r}=\left.\frac{\partial z}{\partial r}\right|_{(0,0,0)}=$ $\frac{2}{L_{i}^{2}}\left(1-\frac{z_{r}^{2} L_{i}^{2}}{2}\right), \varphi_{\theta_{i}}=\left.\frac{\partial z}{\partial \theta_{i}}\right|_{(0,0,0)}=-\frac{2}{L_{i}} \sqrt{1-\frac{z_{r}^{2} L_{i}^{2}}{4}}$, and $\varphi_{z_{i}}=$ $\left.\frac{\partial z}{\partial z_{i}}\right|_{(0,0,0)}=0$.

C. Maximum Allowable Communication Delay

Based on motion analysis, we can obtain the state representations (10) and (11) for CAVs tracking a straight line and a circular curve. Next, we derive the delay requirement.

Theorem 1. The stability of the steering controller at CAV $i$ can be guaranteed if the maximum delay of any V2X link $i$ in the vehicular network satisfies:

$$
\tau_{i}(t) \leq \triangle \tau=\frac{1}{\lambda_{\max }\left(\boldsymbol{D}_{i}\right)},
$$

where $\boldsymbol{D}_{i}=\boldsymbol{C}_{i} \boldsymbol{B}_{i} \boldsymbol{A}_{i} \boldsymbol{A}_{i}^{T} \boldsymbol{B}_{i}^{T} \boldsymbol{C}_{i}^{T}+\boldsymbol{C}_{i} \boldsymbol{B}_{i} \boldsymbol{B}_{i} \boldsymbol{B}_{i}^{T} \boldsymbol{B}_{i}^{T} \boldsymbol{C}_{i}^{T}+2 c \boldsymbol{I}$ with $c>1$ and a positive definitive matrix $\boldsymbol{C}_{i}$ meeting $\boldsymbol{C}_{i}\left(\boldsymbol{A}_{\imath}+\right.$ 
$\left.\boldsymbol{B}_{i}\right)+\left(\boldsymbol{A}_{i}+\boldsymbol{B}_{i}\right)^{T} \boldsymbol{C}_{i}=-\boldsymbol{I}_{3 \times 3}$, and $\lambda_{\max }(\cdot)$ represents the maximum eigenvalue of the corresponding matrix.

Proof: As $\boldsymbol{A}_{i}+\boldsymbol{B}_{i}$ can be verified as Hurwitz stable when the CAV follows a straight line or circular curve, and from Lyapunov theory [17], there always exists a positive definitive matrix $\boldsymbol{C}_{i} \in \mathbb{R}^{3 \times 3}$ so that $\boldsymbol{C}_{i}\left(\boldsymbol{A}_{\boldsymbol{i}}+\boldsymbol{B}_{\boldsymbol{i}}\right)+\left(\boldsymbol{A}_{\boldsymbol{i}}+\boldsymbol{B}_{\boldsymbol{i}}\right)^{T} \boldsymbol{C}_{\boldsymbol{i}}=-\boldsymbol{I}_{3 \times 3}$. Also, similar to the consensus problem considered in [17], we use the following candidate Lyapunov function: $V(\boldsymbol{e})=$ $\boldsymbol{e}^{T} \boldsymbol{C}_{i} \boldsymbol{e}$. Further proof is similar to [2] and is omitted here.

By substituting $\boldsymbol{A}_{i}$ and $\boldsymbol{B}_{i}$ respectively with $\overline{\boldsymbol{A}}$ and $\overline{\boldsymbol{B}}$, or $\hat{\boldsymbol{A}}$ and $\hat{B}$ in Theorem 1, we can obtain the delay requirement to guarantee the stability of the steering controller when tracking a straight line or a circular curve. Note that, as both $\bar{A}$, $\bar{B}, \hat{A}$, and $\hat{B}$ are functions of $L_{i}$, the delay found in (12) is dependent on the value of $L_{i}$. In addition, when a CAV is tracking a road composed of $M$ segments where each segment is either a straight line or circular curve, the maximum delay requirement to guarantee the stability of the steering controller is $\tau_{i}^{\max }=\min \left(\triangle \tau_{1}, \triangle \tau_{2}, \ldots, \triangle \tau_{M}\right), i \in \mathcal{I}$. Based on the delay requirement in Theorem 1, we can determine the reliability of a V2X network for CAVs using V2X-assisted autonomous navigation. Also, Theorem 1 sheds light on how to design the control system, e.g., choosing a proper value of $L_{i}$, to relax the delay requirement and improve the reliability performance of V2X networks. By using this guideline, we propose a joint control and communication system design to solve the problem in (6)-(8) in the next section.

\section{Joint System Design For CAVs}

In order to solve the optimization problem in (6)-(8), we decompose the original problem into two sub-problems: control system design and power allocation for the V2X network. In particular, in the first sub-problem, we form an optimization problem of $L_{i}$ to maximize $\tau_{i}^{\max }$ (to improve the tolerance of the control system to delay), and the sub-problem is solved by using a dual update method. Moreover, after choosing the optimized value of $L_{i}$ from the first sub-problem, we leverage risk tools [18] from financial engineering to determine the power allocation strategy for the communication system.

\section{A. Control System Design}

For the control system design, we can relax the delay requirement and improve the control system's delay tolerance by formulating the following optimization problem:

$$
\begin{aligned}
& \max _{\left\{L_{i}\right\}} \tau_{i}^{\max } \\
& \text { s.t. } L_{\min } \leq L_{i} \leq L_{\max }, \\
& \quad \lambda_{i}^{(m)} \tau_{i}^{\max }-1 \leq 0,1 \leq m \leq M,
\end{aligned}
$$

where $\lambda_{i}^{(m)}$ corresponds to the value of the denominator on the right-hand side in (12) for the $m$-th path segment.

Since the optimization problem in (13)-(15) is not convex, we use the dual update method, introduced in [19], to obtain an efficient sub-optimal solution. In particular, we iteratively update the Lagrange multipliers, and, then, calculate the optimization variables by solving the dual optimization problem. First, we obtain the Lagrange function as:

$$
\mathcal{L}\left(L_{i}, \tau_{i}^{\max }, \nu_{1}, \ldots, \nu_{M+2}\right)=\tau_{i}^{\max }+\sum_{m=1}^{M} \nu_{i}\left(1-\lambda_{i}^{(m)} \tau_{i}^{\max }\right)
$$

$$
+\nu_{M+1}\left(L_{i}-L_{\min }\right)+\nu_{M+2}\left(L_{\max }-L_{i}\right),
$$

where $\nu_{1}, \ldots, \nu_{M+2} \geq 0$ are the Lagrange multipliers. The Lagrange dual function can be thereby expressed as $\max _{L_{i}, \tau_{i}^{\max }} \mathcal{L}\left(L_{i}, \tau_{i}^{\max }, \nu_{1}, \ldots, \nu_{M+2}\right)$. Next, we obtain a subgradient of $\mathcal{L}\left(L_{i}, \tau_{i}^{\max }, \nu_{1}, \ldots, \nu_{M+2}\right)$ as follows:

$$
\begin{aligned}
& \Delta \nu_{m}=1-\Lambda_{\max }^{(i)} \tau_{i}^{*}, 1 \leq m \leq M, \\
& \Delta \nu_{M+1}=L_{i}^{*}-L_{\min }, \Delta \nu_{M+2}=L_{\max }-L_{i}^{*},
\end{aligned}
$$

where $L_{i}^{*}$ and $\tau_{i}^{*}$ are the optimizing variables in Lagrange dual function, and the $\Lambda_{i}^{(m)}$ is the value of $\lambda_{i}^{(m)}$ when $L_{i}=L_{i}^{*}$. The proof of subgradient is similar to the one provided in [19] and is omitted here. We can choose the subgradient method or ellipsoid method to find the optimal dual variables for $\nu_{1}, \ldots, \nu_{M+2}$. Then, the sub-optimal solution of $L_{i}$ and $\tau_{i}^{\max }, i \in \mathcal{I}$, can be determined by solving the dual optimization problem, omitted here due to space limitation. Thus, after solving the optimization problem in (13)-(15), BS can send the headway distance $L_{i}^{*}$ to the receiving CAV in each pair. Then, the CAV can use the $L_{i}^{*}$ in the pure pursuit method to relax the delay requirement and improve the control system's delay tolerance.

\section{B. Financial Risk Based Power Allocation}

After obtaining the optimized control parameter $L_{i}^{*}$, we can optimize the power allocation to maximize the number of reliable V2X links. To solve the problem in (6)-(8) when $L_{i}$ is fixed, we introduce a binary variable $\beta_{i}$ to capture the indicator function in (6) and formulate an equivalent problem:

$$
\begin{aligned}
\max _{\boldsymbol{p},\left\{\beta_{i}\right\}} & \sum_{i \in \mathcal{I}} \beta_{i} \\
\text { s.t. } & \gamma \leq \operatorname{Pr}\left(\tau_{i} \leq \tau_{i}^{\max }\right)+\left(1-\beta_{i}\right), \quad \beta_{i} \in\{0,1\}, i \in \mathcal{I}, \\
& 0 \leq p_{i}(t) \leq p_{i}^{\max }, i \in \mathcal{I} .
\end{aligned}
$$

Here, if $\gamma \geq \operatorname{Pr}\left(\tau_{i} \leq \tau_{\max }\right), i \in \mathcal{I}$, $\beta_{i}$ will be set 0 to meet the constraint (17); otherwise, $\beta_{i}$ will be chosen as 1 to maximize the objective function in (16).

Since Nakagami fading channels are considered for V2X communications, directly deriving the expression of $\operatorname{Pr}\left(\tau_{i} \leq\right.$ $\left.\tau_{i}^{\max }\right)$ is challenging. Alternatively, we consider a risk tool from financial engineering to model the value of $\operatorname{Pr}\left(\tau_{i} \leq \tau_{i}^{\max }\right)$. This is due to the similarity between our optimization problem and risk management problems considered in risk theory. In particular, in risk theory, the objective is to quantify the potential risk and make a decision to minimize the risk of loss, like portfolio optimization problems in [20]. Similarly, our optimization problem seeks to perform power allocation for the wireless system to minimize the number of V2X pairs in which the stability of the control system cannot be guaranteed.

To conduct risk analysis, there are two risk measurement tools commonly used in financial engineering. The first measure is value at risk (VaR), also know as quantile, where the maximal loss is measured with a given probability [18]. Mathematically, $\delta$-VaR of a loss function $\eta$ is defined as $\operatorname{VaR}_{\delta}(\eta)=\inf \{a \in \mathbb{R}: \operatorname{Pr}(\eta \leq a) \geq \delta\}$, where $\delta \in(0,1)$ is the confidence level. To capture the risk distribution beyond the quantile notion of VaR, the CVaR is proposed to capture the mean tail loss and mathematically defined as [20]:

$$
\operatorname{CVaR}_{\delta}(\eta)=\inf _{b \in \mathbb{R}}\left\{\frac{1}{1-\delta} \mathbb{E}\left[[\eta+b]^{+}\right]-b\right\}=\frac{1}{1-\delta} \int_{\delta}^{1} \operatorname{VaR}_{\delta}(\eta) d \delta,
$$


where $[\eta+b]^{+}=\max \{\eta+b, 0\}$. Also, the range of $b$ can be further constrained, i.e., $b \in\left[0, b_{\text {upper }}\right]$, where $b_{\text {upper }}=\operatorname{VaR}_{\delta}(-U(\eta))$ with an upper bound $U(\eta)$ of the loss function $\eta$ [20].

Here, we employ the CVaR to model the value of $\operatorname{Pr}\left(\tau_{i} \leq\right.$ $\left.\tau_{i}^{\max }\right)$ and solve the optimization problem in (16)-(18). In particular, by comparing the delay $\tau_{i}(t)$ with the delay requirement found in Theorem 1, we can define the risk function of $\mathrm{V} 2 \mathrm{X}$ link $i$ when the power allocation strategy is $\boldsymbol{p}$ as follows:

$$
\eta_{i}(\boldsymbol{p})=\left(2^{\frac{S}{\omega \tau_{i}^{\max }}}-1\right)\left(\omega N_{0}+\sum_{j \in \mathcal{I} \backslash i} p_{j}(t) g_{i, j}(t)\right)-p_{i}(t) g_{i, i}(t) \text {. }
$$

Hence, by comparing the value of $\eta_{i}(\boldsymbol{p})$ with 0 , we can determine whether a $\mathrm{V} 2 \mathrm{X}$ communication link $i, i \in \mathcal{I}$, is at risk or not to support the stability of the steering controller. By using the risk function in (19), we can replace the constraint (17) with a $\left(\gamma-1+\beta_{i}\right)-\mathrm{CVaR}$ based constraint expressed as

$$
\inf _{b_{i} \in \mathbb{R}}\left\{\frac{1}{2-\gamma-\beta_{i}} \mathbb{E}\left[\left[\eta_{i}(\boldsymbol{p})+b_{i}\right]^{+}\right]-b_{i}\right\} \leq 0 .
$$

Moreover, based on the sample average method (SAA) [21], we assume that there are $K$ independent and identically distributed (i.i.d.) samples of $\eta_{i}(\boldsymbol{p}), i \in \mathcal{I}$, and an approximation for $\mathbb{E}\left[\left[\eta_{i}(\boldsymbol{p})+t\right]^{+}\right]$can be shown as follows:

$$
\mathbb{E}\left[\left[\eta_{i}(\boldsymbol{p})+b_{i}\right]^{+}\right] \approx \frac{1}{K} \sum_{k=1}^{K} \mathbb{E}\left[\left[\eta_{i, k}(\boldsymbol{p})+b_{i}\right]^{+}\right],
$$

where $\eta_{i, k}(\boldsymbol{p})$ denotes the value of $\eta_{i}(\boldsymbol{p})$ for $k$-th sample. Thus, the optimization problem in (16)-(18) with the CVaR condition can be reformulated as follows:

$$
\begin{array}{ll}
\max _{\boldsymbol{p},\left\{n_{i}^{k}\right\},\left\{b_{i}\right\},\left\{\beta_{i}\right\}} \sum_{i \in \mathcal{I}} \beta_{i} \\
\text { s.t. } & \eta_{i, k}(\boldsymbol{p})+b_{i} \leq n_{i}^{k}, i \in \mathcal{I}, 1 \leq k \leq K, \\
& \sum_{k=1}^{K} n_{i}^{k}-(2-\gamma) K b_{i}+K \beta_{i} b_{i} \leq 0, i \in \mathcal{I}, \\
& n_{i}^{k} \geq 0, i \in \mathcal{I}, 1 \leq k \leq K, \\
& \beta_{i} \in\{0,1\}, 0 \leq p_{i}(t) \leq p_{i}^{\max }, i \in \mathcal{I} .
\end{array}
$$

To solve the approximated problem, we consider $\hat{\beta}_{i}=\beta_{i} b_{i}$. Hence, based on the Big M method introduced in [22], the constraint in (24) can be converted to

$$
\sum_{k=1}^{K} n_{i}^{k}-(2-\gamma) K b_{i}+K \hat{\beta}_{i} \leq 0, i \in \mathcal{I}
$$

$\hat{\beta}_{i} \leq b_{\text {upper }} \beta_{i}, \hat{\beta}_{i} \leq b_{i}, \hat{\beta}_{i} \geq b_{i}-\left(1-\beta_{i}\right) b_{\text {upper }}, \hat{\beta}_{i} \geq 0$. (28) We can observe that the optimization problem with the updated constraints is a mixed integer linear programming (MILP) problem. To solve such problem, we can leverage the wellknown branch and bound algorithm whose details are omitted here due to the space limitation.

By decomposing the original optimization problem in (6) (8) into two sub-problems, we use dual update method and the notion of $\mathrm{CVaR}$ from financial engineering to obtain a suboptimal solution. The solution provides insights how to jointly design the control system and communication network to realize a stable navigation for CAVs.

\section{Simulation Results And Analysis}

For our simulations, we use a Manhattan mobility model in which V2X pairs are uniformly distributed over the space
TABLE I

SIMULATION PARAMETERS.

\begin{tabular}{|c|c|}
\hline Parameter & Value \\
\hline Simulation area & $210 \times 210 \mathrm{~m}^{2}$ \\
\hline Transmission power $p^{\max }$ (V2V, V2P, V2I pairs) & $27,24,28 \mathrm{dBm}$ \\
\hline Building breadth, street width & $100 \mathrm{~m}, 10 \mathrm{~m}[12]$ \\
\hline Bandwidth $\omega$, noise spectral density $N_{0}$ & $20 \mathrm{MHz}[2],-174 \mathrm{dBm} / \mathrm{Hz}[12]$ \\
\hline Speed range & $(5,20) \mathrm{m} / \mathrm{s}$ \\
\hline Distance range for V2V, V2P, and V2I pairs & $(10,20),(2,10),(10,40) \mathrm{m}$ \\
\hline Curvature range & $(0,1 / 2)[10]$ \\
\hline Nakagami parameter, path loss exponent & $3,4[13]$ \\
\hline Packet size $S$ & $(100,5000) \mathrm{bits}$ \\
\hline Minimum \& maximum headway $L_{\min } \& L_{\max }$ & $4,10 \mathrm{~m}$ \\
\hline Time slot duration $T$ & $10 \mathrm{~ms}[12]$ \\
\hline Number of samples $K$ & 100 \\
\hline
\end{tabular}
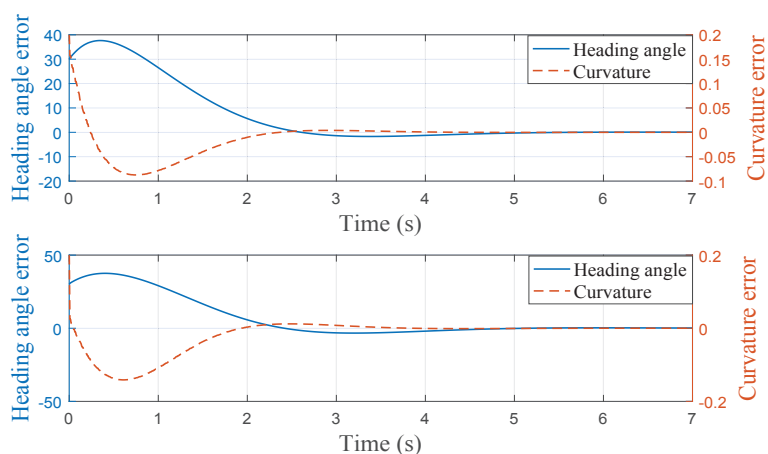

Fig. 3. Validation of the maximum tolerable delay found in Theorem 1. The top figure is for the straight line and the bottom one is for the circular curve.

outside buildings. Also, each CAV will follow a sequence of straight lines and circular curves and its speed is assumed in a bounded range, shown in Table I. Also, other simulation parameters are summarized in Table I.

We first corroborate our analytical results on delay requirements to guarantee the stability of the steering controller. In particular, when $L=5 \mathrm{~m}$ and $z_{r}=0.2$, we can find that the delay requirements for vehicles driving on the straight line and the circular curve are $16.1 \mathrm{~ms}$ and $18.2 \mathrm{~ms}$. Thus, we model the uncertainty of the V2X links as a time-varying delay in the range $(0,16.1 \mathrm{~ms})$ and $(0,18.2 \mathrm{~ms})$, respectively, when the CAV is tracking a straight line and a circular curve. Initially, the CAV is driving with randomly selected heading angle and curvature. As shown in Fig. 3, both curvature and heading angle errors for vehicles tracking straight lines and circular curves will converge to 0 (a similar result is observed for the lateral distance error and is omitted due to space limitation). In particular, the CAV's controller can reach the target point in a short period of time, i.e., $5 \mathrm{~s}$, guaranteeing the stability of the steering controller. Clearly, the delay requirements, found by Theorem 1, can guarantee the stability of the controller to track both straight and circular paths.

Fig. 4 shows how the percentage of reliable V2X pairs changes as the packet size $S$ increases in a network with a total of $I=120 \mathrm{~V} 2 \mathrm{X}$ pairs. We also compare the performance of our proposed joint design with three baseline systems. In particular, the first baseline is the system with optimized headway distance $L_{i}, i \in \mathcal{I}$, for the control system, and the transmission power for each pair is randomly selected in the range shown in Table I. For the second baseline, the system is with optimized power allocation for V2X communications, while the headway distance for each pair is randomly selected 


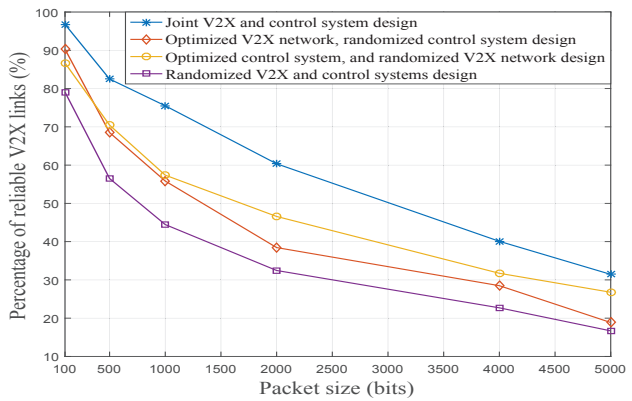

Fig. 4. Reliability performance versus packet size.

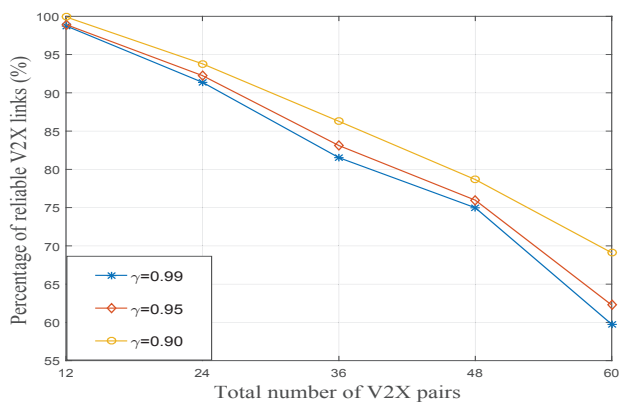

Fig. 5. Reliability performance versus total number of V2X pairs.

in the range shown in Table I. For the third baseline system, both headway distance and transmission power are randomly selected in the bounded ranges of Table I. As shown in Fig. 4, we can observe that, as the packet size $S$ increases, the percentage for reliable V2X pairs decreases. This is due to the fact that a large-sized packet will increase the V2X transmission delay, leading to a lower reliability for the vehicular communication. Moreover, when $S=5000$ bits, the percentage of the reliable V2X links for the system with the optimized power allocation and the system with random V2X and control system design is $19 \%$ and $18 \%$, respectively. However, the performance for the joint design strategy can be as much as $32 \%$ which is approximately a $70 \%$ improvement compared with the baseline schemes that optimize the communication and control systems independently.

Fig. 5 shows the percentage of reliable V2X pairs for traffic scenarios with different number of V2X links. Also, we compare the reliability performance for systems with different target reliability thresholds. As observed in Fig. 5, when the total number of $\mathrm{V} 2 \mathrm{X}$ links increases, the percentage of reliable V2X pairs will decrease. This is because, if the traffic density increases, the receiving CAV will encounter more interference generated by other links. As a result, the SINR and the system throughput will decrease, leading to a lower reliability for the V2X network. In addition, Fig. 5 shows that a system with higher target reliability threshold will have a lower percentage of reliable V2X pairs in the network. This is because, with higher target reliability, fewer V2X pairs can meet such requirement, leading to a lower percentage.

\section{CONCLUSION}

In this paper, we have proposed a novel joint control system and V2X wireless communication design framework that enables CAVs to leverage V2X connectivity for autonomous navigation (i.e., path tracking). Based on the proposed joint de- sign strategy, we have determined the maximum transmission delay which can prevent the instability of the CAV's control system. We have also decomposed the joint design problem into two sub-problems: control system design and power allocation for communication network. To find an efficient solution to these two sub-problems, we have utilized the dual update method and the risk notion of CVaR from financial engineering. Simulation results have validated our theoretical results and shown that the proposed joint design can improve the number of reliable $\mathrm{V} 2 \mathrm{X}$ pairs by as much as $70 \%$ compared to a baseline that optimizes the communication and control systems independently.

\section{REFERENCES}

[1] R. Hussain and S. Zeadally, "Autonomous cars: Research results, issues and future challenges," IEEE Commun. Surveys Tuts., to appear, 2018.

[2] T. Zeng, O. Semiari, W. Saad, and M. Bennis, "Joint communication and control for wireless autonomous vehicular platoon systems," arXiv preprint, 2018. [Online]. Available: arxiv.org/abs/1804.05290

[3] O. Semiari, W. Saad, M. Bennis, and M. Debbah, "Integrated millimeter wave and sub- $6 \mathrm{GHz}$ wireless networks: A roadmap for joint mobile broadband and ultra-reliable low-latency communications," IEEE Wireless Communications, pp. 1-7, 2019.

[4] D. J. Fagnant and K. Kockelman, "Preparing a nation for autonomous vehicles: opportunities, barriers and policy recommendations," Transportation Research Part A: Policy and Practice, vol. 77, pp. 167-181, Jul. 2015.

[5] G. Jagadeesh, T. Srikanthan, and X. Zhang, "A map matching method for GPS based real-time vehicle location," The Journal of Navigation, vol. 57, no. 3, pp. 429-440, Aug. 2004.

[6] K. Golestan, F. Sattar, F. Karray, M. Kamel, and S. Seifzadeh, "Localization in vehicular ad hoc networks using data fusion and V2V communication," Computer Communications, vol. 71, pp. 61-72, Nov. 2015.

[7] G. Soatti, M. Nicoli, N. Garcia, B. Denis, R. Raulefs, and H. Wymeersch, "Implicit cooperative positioning in vehicular networks," IEEE Transactions on Intelligent Transportation Systems, to appear, 2018.

[8] L. Le, A. Festag, R. Baldessari, and W. Zhang, "V2x communication and intersection safety," in Advanced Microsystems for Automotive Applications 2009. Springer, 2009, pp. 97-107.

[9] A. Ghods, S. Severi, and G. Abreu, "Localization in V2X communication networks," in Proc. of IEEE Intelligent Vehicles Symposium, Gothenburg, Sweden, Jun. 2016.

[10] G. Heredia and A. Ollero, "Stability of autonomous vehicle path tracking with pure delays in the control loop," Advanced Robotics, vol. 21, no. $1-2$, pp. 23-50, Jan. 2007.

[11] T. Zeng, M. Mozaffari, O. Semiari, W. Saad, M. Bennis, and M. Debbah, "Wireless communications and control for swarms of cellular-connected UAVs," in Proc. of IEEE Asilomar Conference on Signals, Systems, and Computers, Pacific Grove, CA, USA, Oct. 2018.

[12] M. I. Ashraf, M. Bennis, C. Perfecto, and W. Saad, "Dynamic proximityaware resource allocation in vehicle-to-vehicle (V2V) communications," in Proc. of IEEE Globecom Workshops, Washington, DC, USA, Dec. 2016.

[13] R. He, A. F. Molisch, F. Tufvesson, Z. Zhong, B. Ai, and T. Zhang, "Vehicle-to-vehicle propagation models with large vehicle obstructions," IEEE Trans. Intell. Transp. Syst., vol. 15, no. 5, pp. 2237-2248, Oct.

[14] M. Buehler, K. Iagnemma, and S. Singh, The 2005 DARPA grand challenge: the great robot race. Springer, 2007, vol. 36.

[15] M. Bennis, M. Debbah, and H. V. Poor, "Ultra-reliable and low-latency wireless communication: Tail, risk and scale," Proceedings of the IEEE vol. 106 , no. 10 , pp. $1834-1853$, Oct. 2018.

[16] T. P. Nguyen and I. Debled-Rennesson, "Decomposition of a curve into arcs and line segments based on dominant point detection," in Proc. of Scandinavian Conference on Image Analysis, Ystad, Sweden, May 2011.

[17] K. Liu, G. Xie, and L. Wang, "Consensus for multi-agent systems under double integrator dynamics with time-varying communication delays," International Journal of Robust and Nonlinear Control, vol. 22, no. 17, pp. 1881-1898, Sept. 2012

[18] D. Duffie and J. Pan, "An overview of value at risk," Journal of derivatives, vol. 4, no. 3, pp. 7-49, Jan. 1997.

[19] W. Yu and R. Lui, "Dual methods for nonconvex spectrum optimization of multicarrier systems," IEEE Trans. Commun., vol. 54, no. 7, pp. 13101322, Jul. 2006

[20] L. J. Hong, Z. Hu, and L. Zhang, "Conditional value-at-risk approximation to value-at-risk constrained programs: A remedy via monte carlo," INFORMS Journal on Computing, vol. 26, no. 2, pp. 385-400, Feb. 2014.

[21] A. Shapiro, D. Dentcheva, and A. Ruszczyński, Lectures on stochastic programming: modeling and theory. SIAM, 2009.

[22] I. Griva, S. G. Nash, and A. Sofer, Linear and nonlinear optimization. Siam, 2009, vol. 108. 